\title{
Hydrodynamics of ring nebulae: magnetic vs. non-magnetic hydro-models
}

\author{
Guillermo García-Segura ${ }^{1}$, Norbert Langer ${ }^{2}$, Michał Różyczka ${ }^{3}$, \\ José Franco ${ }^{4}$, and Mordecai-Mark Mac Low ${ }^{5}$ \\ ${ }^{1}$ Instituto de Astronomía-UNAM, \\ Apdo Postal 877, Ensenada, 22800 Baja California, Mexico \\ ${ }^{2}$ Institut für Physik, Universität Potsdam, D-14415 Potsdam, Germany \\ ${ }^{3}$ N. Copernicus Astronomical Center, \\ Bartycka 18, 00-716 Warszawa, Poland \\ ${ }^{4}$ Instituto de Astronomía-UNAM, \\ Apdo Postal 70-264, 04510 México D.F., Mexico \\ ${ }^{5}$ Max-Planck-Institut für Astronomie, \\ Königstuhl 17, D-69117 Heidelberg, Germany
}

\begin{abstract}
The formation of ring nebulae and their shapes is studied by numerical simulations. The old problem of the origin of the nebular shape is addressed. Two proposed mechanisms are discussed: stellar rotation and stellar magnetic fields, or a combination of both. Two and three-dimensional hydrodynamical and magneto-hydrodynamical simulations indicate that these processes are equally efficient in driving elliptical nebulae such as those surrounding WolfRayet stars, like NGC 6888 around WR 136. However, bipolar nebulae are better reproduced by rotation.
\end{abstract}

\section{Introduction}

It is commonly accepted that the formation of circumstellar nebulae involves the action of at least two interacting stellar winds, for both planetary nebulae (Kwok, Purton \& Fitzgerald 1978; Kwok 1982, 1994; Kahn 1983; Balick 1987; Aller 1993) and ring nebulae around Pop. I massive stars (García-Segura \& Mac Low 1995). Chu (1993) provided extensive evidence that PNe and ring nebulae around massive stars are not only similar in their morphologies. For massive stars that evolve through red supergiant, or luminous blue variable phases towards a Wolf-Rayet phase, the final WR fast wind would sweep up the slow RSG or LBV wind and form a bubble of stellar material, reminiscent of the two-wind formation of PNe.

It has been shown by Langer (1997) and by García-Segura \& López (1999) that both LBVs and AGB stars reach the $\Omega$-limit of critical rotation, independently of the actual stellar rotation rate. García-Segura et al. (1997) and Langer et al. (1999) have shown that the result is a slow, massive, equatorially confined outflow, which may be inflated to a double lobe structure by a fast spherically symmetric wind later on. The striking similarity of the shapes of some $\mathrm{PNe}$ and LBV nebulae - e.g., compare the Hourglass Nebula MyCn 18 with the Homunculus Nebula surrounding $\eta$ Carinae - is certainly in support of the view 
that they have a common underlying physical principle. This is in line with an earlier suggestion by Calvet \& Peimbert (1983). In such a case the main cause of bipolarity would be stellar rotation.

Asymmetries of the precursor AGB wind are not the only factor responsible for the shaping of PNe. Chevalier \& Luo (1994) obtained aspherical nebulae produced by a rotating star with a magnetized fast wind. The aspherical shapes were due solely to the tension of the toroidal magnetic field. Recent MHD simulations by Różyczka \& Franco (1996) (2-dimensional, cylindrical calculations) and by García-Segura et al. (1999) (2-dimensional, spherical calculations) have shown that magnetic effects may also be responsible for the generation of collimated flows (jets) observed in some nebulae. The 3-D computations performed by García-Segura (1997) show that the behavior of the gas is similar to those computed in 2-dimensions, with the only difference that the collimated gas can be subject to kink instabilities. Thus, the formation of jets and ansae are confirmed in 3-dimensional, axis-free, calculations.

The first question that we have to answer concerning the formation of ring nebulae is how and where are ring nebulae formed in the HR diagram?. We have made some progress in answering such a question by directly coupling stellar evolution calculations with hydrodynamical calculations for the ejected gas. These calculations include the transitions $\mathrm{O} \rightarrow \mathrm{RSG} \rightarrow \mathrm{WR}$ (see GarcíaSegura et al. 1996a) which reproduces to first order the nebula NGC 6888, and the transitions $\mathrm{O} \rightarrow \mathrm{LBV} \rightarrow \mathrm{WR}$ (see García-Segura et al. 1996b) which reproduces to first order the nebula around the LBV P Cygni.

The second question is what physical reason is shaping the nebula? Usually, two answers have been given in the literature: a) density gradients in the winds and $b$ ) the effects of magnetic fields. We here explore those solutions for single stars, for which the solution is provided (a) by stellar rotation, and (b) by the stellar magnetic field.

\section{The effects of stellar rotation: the Omega-limit}

In the Hertzsprung-Russell diagram, LBV stars like $\eta$ Car lie close to the upper borderline of temperature and luminosity beyond which no normal stars are observed (Humphreys \& Davidson 1979), near the location of the Eddingtonlimit.

As Figure 1 shows, a massive star expanding at constant luminosity $L$ would - in the non-rotating case - reach the Eddington-limit due to an opacity increase in its surface layers. The Eddington-limit is reached when $\Gamma=L / L_{\mathrm{Edd}}=$ 1, where the Eddington luminosity $L_{\mathrm{Edd}}=4 \pi c G M / \kappa$, with $c$ the speed of light, $M$ and $L$ the mass and luminosity of the star, and $\kappa$ the opacity at the stellar surface. Note that when $\Gamma \rightarrow 1$, the escape velocity $v_{\text {esc }}=[(1-\Gamma) 2 G M / R]^{1 / 2} \rightarrow 0$. This implies a very slow wind velocity $(\sim 0)$ at the Eddington-limit. Thus, when a star passes through a close approach to the Eddington-limit, its stellar wind will follow a fast-slow-fast sequence.

The critical rotational velocity, where the combination of radiation pressure and centrifugal force exceeds surface gravity at the equator, given by $v_{\text {crit }}=[(1-$ $\Gamma) G M / R]^{1 / 2}$, has the same behavior as $v_{\text {esc }}$, i.e., when $\Gamma \rightarrow 1, v_{\text {crit }} \rightarrow 0$. However, the rotational velocity $v_{\text {rot }}$ is always finite. That is, critical rotation occurs before 


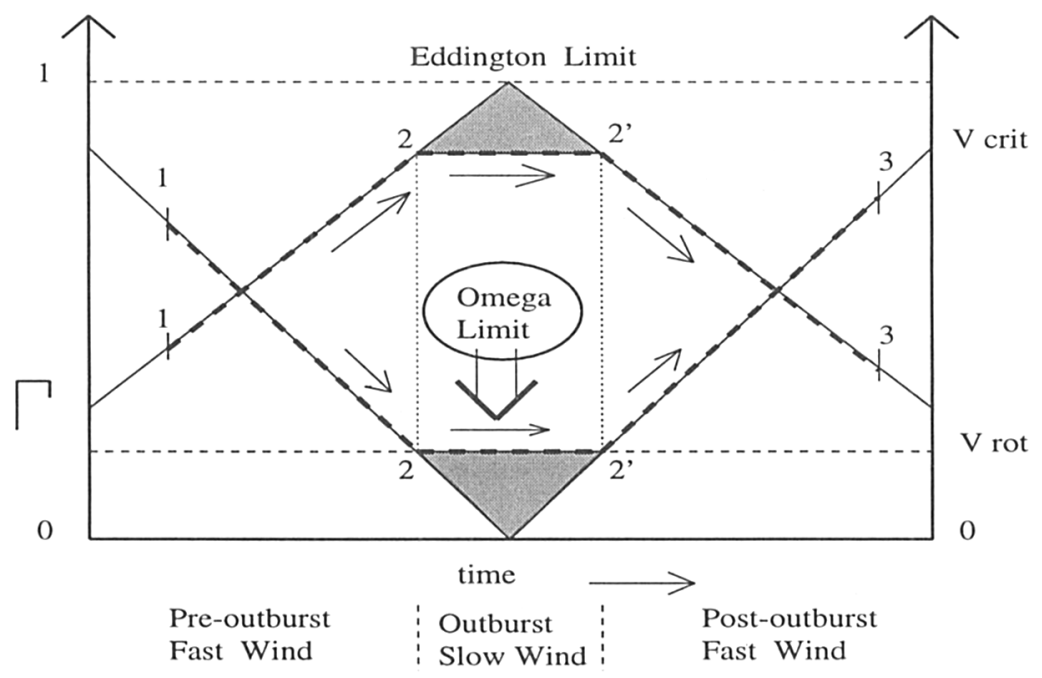

Figure 1. Time evolution of the Eddington factor $\Gamma$ and the critical rotation velocity $v_{\text {crit }}$. For simplicity, we assumed a constant equatorial rotational velocity $v_{\text {rot }}$ (dashed line). As discussed in the text, the outburst (2-2') will occur before the star reaches its Eddington-limit (dashed line), at the Omegalimit.

the Eddington-limit is reached. This is the so called Omega-limit (Langer 1997). Winds with mass loss rates large enough to halt and reverse the stellar expansion are thought to occur when $\Omega=v_{\text {rot }} / v_{\text {crit }}$ approaches unity (Friend \& Abbott 1986; Bjorkman \& Cassinelli 1993). Thus, outflows will always occur before the star reaches its Eddington-limit, acting to prevent further expansion.

\section{The effects of stellar magnetic fields: the magnetic tension}

The magnetic field in an outflowing wind can be described by two components. The toroidal component, $B_{\phi}$, is set using the equations for a rotating star:

$$
B_{\phi}=B_{\mathrm{s}} \frac{v_{\mathrm{rot}}}{v_{\infty}(\theta)}\left(\frac{r_{\mathrm{s}}}{r}\right)^{2}\left(\frac{r}{r_{\mathrm{s}}}-1\right) \sin \theta
$$

where $B_{\mathrm{s}}$ and $r_{\mathrm{s}}$ are the magnetic field strength at the stellar surface and the stellar radius, respectively, with the inclusion of the wind-compressed field effects (see Ignace et al. 1998).

The latter equation is a generalization of the standard formula for $B_{\phi}$ in the equatorial $\left(\theta=90^{\circ}\right)$ plane (see e.g., Parks 1991). $B_{\phi}(\theta)$ decreases with decreasing $\theta$ due to an increase in $v_{\infty}$, and the sinusoidal function is included to cancel entirely the toroidal field component at the $\theta=0^{\circ}$ axis. This generalization is obviously arbitrary but, as Różyczka \& Franco (1996) have shown, the precise form of the off-equator distribution of the field is unimportant provided that the 
field is sufficiently strong to cause an appreciable deformation of the shocked wind region. The radial field component can be neglected (see Chevalier \& Luo 1994), so the used field configuration obeys $\nabla \cdot B=0$.

Our models are identified by the dimensionless parameter $\sigma$, which is the ratio of the magnetic field energy density to the kinetic energy density in the fast wind (Chevalier \& Luo 1994)

$$
\sigma=\frac{B^{2}}{4 \pi \rho v_{\infty}^{2}}=\frac{B_{\mathrm{s}}^{2} r_{\mathrm{s}}^{2}}{\dot{M} v_{\infty}}\left(\frac{v_{\mathrm{rot}}}{v_{\infty}}\right)^{2}
$$

See García-Segura et al. (1999) for some examples.

The toroidal field carried by the fast wind can constrain the motion of the flow and an elliptical nebula is produced even if the slow wind is spherically symmetric. The interaction of the two winds generates a hot wind-shocked region where the tension of the compressed $B$-field generates an important new feature: a pair of flows are induced (one at each hemisphere), that move the gas toward the polar regions and create a pair of collimated outflows at the poles (Różyczka \& Franco 1996). The basic features of the collimation mechanism are the following. First, the outer part of the magnetized shocked wind region becomes magnetically rather than thermally supported (i.e., the energy density of the compressed field grows larger than the thermal energy density). Then, the tension of the toroidal field slows down the expansion in the direction perpendicular to the symmetry axis (but the expansion in the direction parallel to the axis proceeds unimpeded) and, given that the intensity of the toroidal field decreases toward the axis, a magnetic pressure gradient in the $z$-direction exists. As a consequence and because of the hoop stress of the toroidal field, a flow in the shocked wind region toward the symmetry axis is initiated and maintained, leading to the formation of stagnation regions at the axis and to the formation of jets. Note that this mechanism works out similarly to that of magnetic confinement experiments in plasma laboratories (e.g., Tokamaks, plasma guns). The gas that arrives at the polar regions of the nebula can form relatively dense blobs, depending of the radiative cooling conditions (García-Segura \& López 1999), which may be identified with ansae observed in nebulae like NGC 6905.

\section{Bipolar ring nebulae}

$\eta$ Car is the prototype of bipolar LBV ring nebula. Using the idea described in section 2, we simulate the LBV outburst phenomenon by allowing the star to pass through three successive stages - pre-outburst, outburst and post-outburst - with correspondingly different stellar winds. The simulations were performed with the hydrodynamic code ZEUS. See García-Segura et al. (1997) for further details. To include the effects of stellar rotation on the winds, we use the analytic model of Bjorkman \& Cassinelli (1993), with stellar parameters appropriate to $\eta$ Car. We compute the hydrodynamic evolution of the circumstellar gas, starting our computations at the beginning of the giant outburst in 1840 A.D., and run them until 1996 A.D. We show in Fig. 2 a model at 1996 A.D. Our main results depend only on our basic outburst scenario: the star is close to its Eddington-limit during outburst (1840-1860 A.D., 2-2' in Fig. 1), but is hotter, 

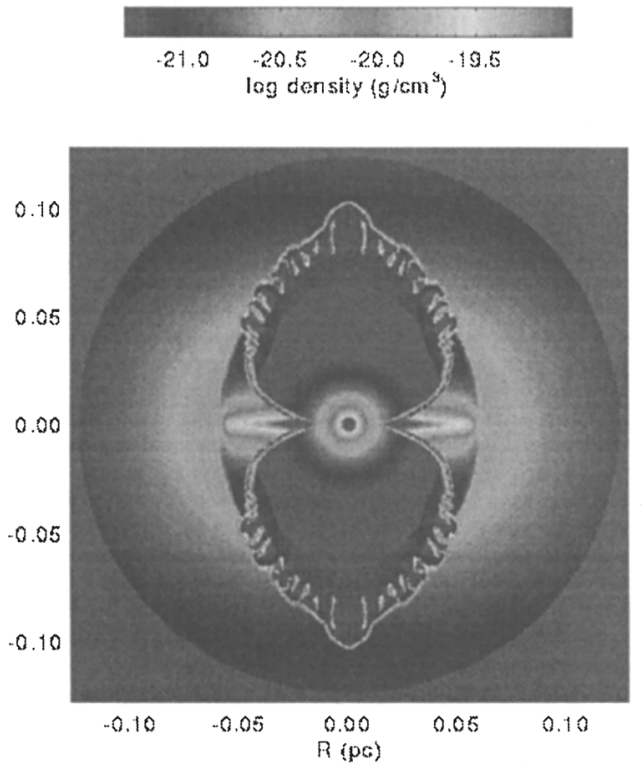

Figure 2. Hydrodynamical model for $\eta$ Car at 1996 A.D.

more compact, and thus further away from the Eddington-limit before and after outburst (1,3 in Fig. 1). The smaller value of $\Gamma$ before and after the outburst has two consequences: larger wind velocities due to larger escape velocities and smaller values of $\Omega$ leading to more spherical winds.

Our outburst scenario leads to a characteristic distribution of the circumstellar gas. The initial fast wind (1 in Fig. 1) forms the background for the subsequent development of the nebula. During outburst (1840-1860 A.D., 2-2'), the wind becomes slow and dense, and the stellar rotation concentrates it toward the equatorial plane (Bjorkman \& Cassinelli 1993; Cassinelli et al. 1995). When the final fast wind (3 in Fig. 1) starts, it sweeps up the dense wind from the outburst into a thin, radiatively cooled shell that fragments due to dynamical instabilities (García-Segura et al. 1996a,b). The shell expands more easily into the lower density wind at the poles, producing a double-lobed structure, as shown in Fig. 2.

\section{Elliptical ring nebulae}

NGC 6888, around WR 136, is the prototype of elliptical WR ring nebulae. In order to explain this type of morphology, we have made an interesting experiment, which gives as a result an ambiguity. With the approaches described in sections 2 and 3, four examples of HD and MHD numerical calculations are shown in Fig. 3. 

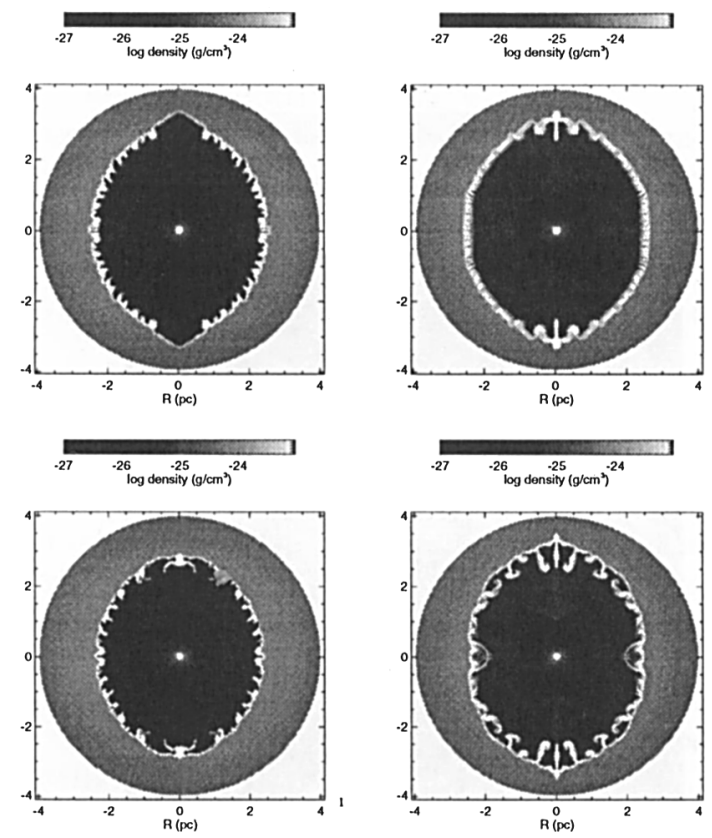

Figure 3. Hydrodynamical (left) vs. magneto-hydrodynamical (right) simulations for elliptical WR ring nebulae. Botton panels have twice numerical resolution.

The left panels in Fig. 3 are HD simulations which have a rotating RSG slow wind with $\Omega=0.30 \Omega_{\mathrm{th}}$, being $\Omega_{\mathrm{th}}$ the threshold value for the formation of a wind-compressed disk (Bjorkman \& Cassinelli 1993; Ignace et al. 1996). The right panels are MHD simulations in which $\sigma=3.5 \times 10^{-4}$. The resolution in the lower panels has been increased by a factor of two to avoid numerical dependences (upper panels are simulations with $200 \times 180$ while lower panels have $400 \times 360$ ). The result is that both physical approaches, the hydrodynamical and the magneto-hydrodynamical simulations give almost identical elliptical shapes.

\section{Summary}

Our model moves well beyond other recent hydrodynamic models for LBV nebulae by directly tying the outburst - the visual maximum in the LBV light curves - to the equatorially confined slow wind. Earlier models (Nota et al. 1995; Frank et al. 1995) concluded that a strong equatorial density enhancement must have existed before the outburst occurred. In contrast, we obtain self-consistently from our model not only the two lobes, with their small-scale structure, but also the equatorial density enhancement, and relate both to the evolutionary state of the star.

We find that our proposed scenario for giant LBV outbursts in rotating, massive, single stars leads naturally to a circumstellar gas distribution strikingly similar to the Homunculus nebula of $\eta$ Car. This is so because stars with 
a finite rotation velocity arrive at critical rotation when they approach their Eddington-limit. The clear bipolar nature of virtually all LBV nebulae (Nota et al. 1995), as well as the progressive, more-spherical morphology that LBV nebulae observations reveal inside of an evolutionary dynamical sequence (Nota \& Clampin 1997), lend strong support to this scenario.

Bipolar shapes (i.e., with an equatorial waist) are better explained by rotational effects. On the other hand, elliptical WR ring nebulae such as the case of NGC 6888 around WR 136 can be explained by either HD or MHD models. Because $\sigma \propto\left(\dot{M} v_{\infty}^{3}\right)^{-1}$, more powerful winds translate into lower $\sigma$ (unless $B_{s}$ correlates with the mass of the star). Lower efficiency of magnetic collimation means increased percentage of ellipticals generated by purely hydrodynamical effects. However, the surface magnetic field of WR stars is unknown, and from there elliptical shapes cannot be explained yet without ambiguity.

Acknowledgments. We thank J. Bjorkman for useful comments and for providing us with his code. We thank Michael L. Norman and the Laboratory for Computational Astrophysics for the permission to use ZEUS-3D. The computations were performed at the Supercomputer Center of the Universidad Nacional Autónoma de México, Rechenzentrum Garching of the Max-Planck-Gesellschaft and at Instituto de Astronomía-UNAM. This work was partially supported by DGAPA-UNAM grant IN130698, CONACyT grant 400354-5-4843E, by the Deutsche Forschungsgemeinschaft through grants La 587/15-1 and La 587/16-1, and by a R\&D CRAY research grant. MR acknowledges partial support from the Committee for Scientific Research through the grants 2P 30401707 and 2P.03D.004.13.

\section{References}

Aller, L.H. 1993, in: R. Weinberger \& A. Acker (eds.), Planetary Nebulae, Proc. IAU Symp. No. 155 (Dordrecht: Kluwer), p. 1

Balick, B. 1987, AJ 94, 671

Bjorkman, J.E., Cassinelli, J.P. 1993, ApJ 409, 429

Calvet, N., Peimbert, M. 1983, RevMexAA 5, 319

Cassinelli, J.P., Ignace, R., Bjorkman, J.E. 1995, in: K.A. van der Hucht \& P.M. Williams (eds.), Wolf-Rayet Stars: Binaries, Colliding Winds, Evolution, Proc. IAU Symp. No. 163 (Dordrecht: Kluwer) p. 191

Chevalier, R.A., Luo, D. 1994, ApJ 421, 225

Chu, Y.-H. 1993, in: R. Weinberger \& A. Acker (eds.), Planetary Nebulae, Proc. IAU Symp. No. 155 (Dordrecht: Kluwer), p. 139

Frank, A., Balick, B., Davidson, K. 1995, ApJ 441, L77

Friend, D.B., Abbott, D.C. 1986, ApJ 311, 701

García-Segura, G. 1997, ApJ 489, L189

García-Segura, G., Langer, N., Różyczka, M., Franco, J. 1999, ApJ 517, 2

García-Segura, G., Langer, N., Mac Low, M.-M. 1997, in: A. Nota \& H. Lamers (eds.), Luminous Blue Variables: Massive Stars in Transition, ASP-CS 120, 332

García-Segura, G., Mac Low, M.-M. 1995, ApJ 455, 160

García-Segura, Mac Low, M.-M., Langer, N. 1996a, A\&A 316, 133

García-Segura, G., Langer, N., Mac Low, M.-M. 1996b, A\&A 305, 229

García-Segura, G., López, J.A. 1999, in preparation 
Humphreys, R. M., Davidson, K. 1979, ApJ 232, 409

Ignace, R., Cassinelli, J.P., Bjorkman, J.E. 1996, ApJ 459, 671

Ignace, R., Cassinelli, J.P., Bjorkman, J.E. 1998, ApJ 505, 910

Kahn, F.D. 1983, in: D.R. Flower (ed.), Planetary Nebulae, Proc. IAU Symp. No. 103, (Dordrecht: Kluwer), p. 305

Kwok, S., Purton, C.R., Fitzgerald, P.M. 1978, ApJ 219, L125

Kwok, S. 1982, ApJ 258, 280

Kwok, S. 1994, PASP 106, 344

Langer, N. 1997, in: A. Nota \& H. Lamers (eds.), Luminous Blue Variables: Massive Stars in Transition, ASP-CS 120, 35

Langer, N., García-Segura, G., Mac Low, M.-M. 1999, ApJL submitted

Nota, A., Livio M., Clampin M., Schulte-Ladbeck R. 1995, ApJ 448, 788

Nota, A., Clampin, M. 1997, in: A. Nota \& H. Lamers (eds.), Luminous Blue Variables: Massive Stars in Transition, ASP-CS 120, 315

Parks, G.K. 1991, in: Physics of Space Plasmas: An Introduction (New York: AddisonWesley)

Różyczka, M., Franco, J. 1996, ApJ 469, L127

\section{Discussion}

Dyson: UV absorption data show that the material in RCW 58 is clumpy. How would that affect your models?

Garcia-Segura: The clumps can be produced either in the instabilities of the sweptup shells or in the wind itself. Clumps generally enhance the radiative cooling. Your models of mass-loading flows will then be a good solution.

Marchenko: This is not a question, this is rather a suggestion. As long as you have some nebulae with dust, and the dust generation is very sensitive to the presence of a weak magnetic field, considering the presence of the dust might place a firm upper limit on the magnetic field strength.

Schulte-Ladbeck: You said you need info on surface $B$-fields. Can you work your way back from the nebular field to the surface field it originated from? For instance, the IR linear polarization of the Homunculus around $\eta$ Car requires the presence of a weak field in the Homunculus.

Garcia-Segura: Yes, we can do it, in principle, by using a complete model for the magnetized wind.

Zhekov: Why is considering a strong magnetic field to shape the nebulosity physically more reasonable than simply assuming that the outer (slower) wind was a-spherical? We don't know anything about the strength of the surface magnetic field. On the other hand, we need a strong field in order to have the a-spherical shape. But we do not need magnetically-driven winds for the hot stars, since the radiation-driven winds theory seems to be OK for these stars.

Garcia-Segura: I have done this study just to test what the HD and the MMD can offer. I really don't know what is the better solution. I never said that the MMD solution is more reasonable. In fact, for massive stars, I am in favour of the HD, rotating models.

Gayley: How important for your rotation model is the WCD paradigm? If the radiative flux aligns with the effective gravity, as it must at the surface, then the radiation force won't be central and won't conserve angular momentum. Is the radiative force important? 
Garcia-Segura: The WCD paradigm is important in line-driven winds. We don't know yet how important it is in continuum-driven winds, which is the case that we are mainly computing.

Leitherer: You are suggesting that some (or even many) WR stars are associated with nebulosities ejected during a RSG phase. Therefore we should expect to observe such ejectae directly around RSG as well. What is the observational evidence?

Garcia-Segura: Stencel et al. ('88, '89) examined IRAS observations of 111 RSG stars. $25 \%$ is spatially resolved by IRAS at $60 \mu \mathrm{m}$ and has extended circumstellar shells $(<2.5 \mathrm{pc})$.

Moffat: You and Tony Marston talked about RSGs in the mass range $30-40 \mathrm{M}_{\odot}$. Yet Massey recently surveyed Local Group galaxies for RSG's. In his rather complete survey, he found that the most luminous (hence massive) RSG's have initial masses in the range $15-20 \mathrm{M}_{\odot}$, depending on the metallicity. Does this mean that your star-wind phase can only be due to LBV's?

Langer: Those masses are quite uncertain. The error bar is quite large.

Dougherty: A comment: in the colliding-wind systems where we detect non-thermal emission, we can estimate the equipartition magnetic field from the non-thermal luminosity. For WR 147, in the collision zone $B_{e q}$ is a few milligauss, which gives a surface field on the O-star similar to those predicted by Barker (1986). Furthermore, the fact that we detect $1.6 \mathrm{GHz}$ emission, in some WR stars, implies that $B \simeq 1$ milli-Gauss, otherwise the Razin effect would suppress the emission at this frequency.

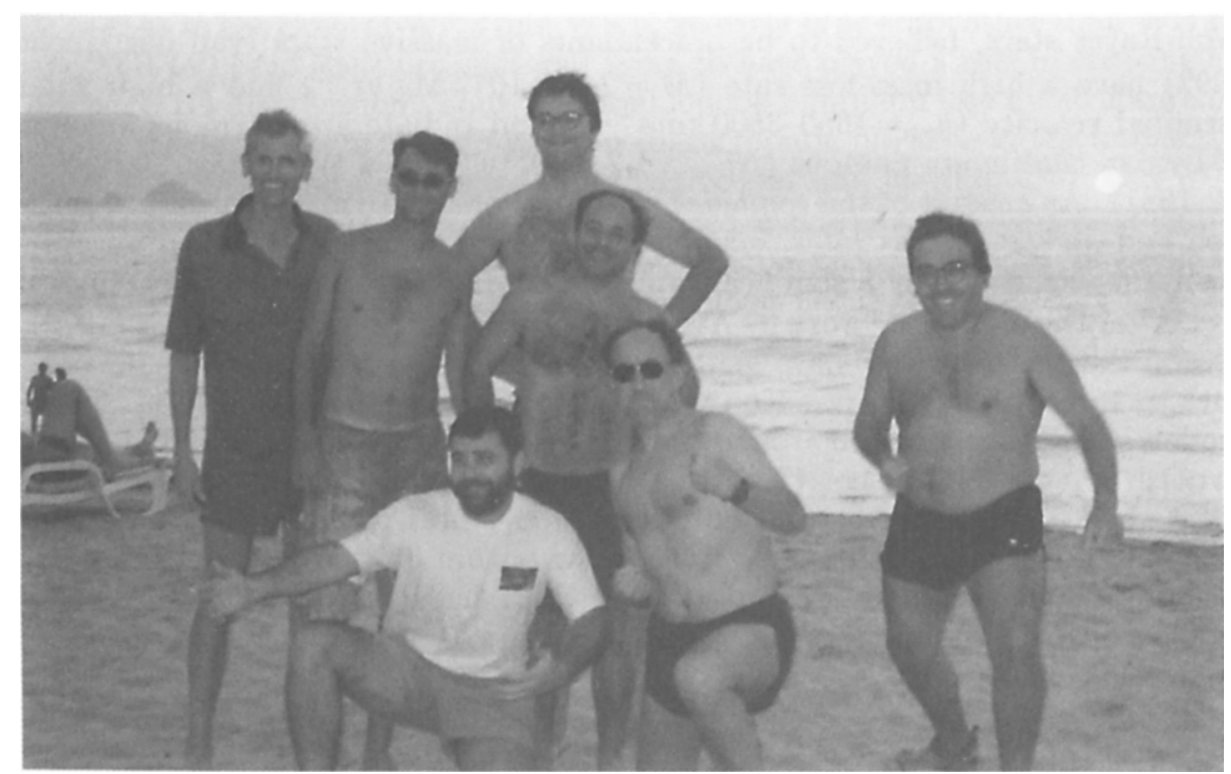

